\title{
Evaluation of severity score-guided approaches to macrolide use in community-acquired pneumonia
}

\author{
Aran Singanayagam ${ }^{1}$ Stefano Aliberti (ib) ${ }^{2,3}$, Catia Cillóniz ${ }^{4}$, Antoni Torres ${ }^{4}$, \\ Francesco Blasi ${ }^{2,3}$ and James D. Chalmers ${ }^{5}$
}

Affiliations: ${ }^{1}$ Dept of Respiratory Medicine, St. Mary's Hospital, Imperial College London, London, UK. ${ }^{2}$ Dept of Pathophysiology and Transplantation, Universita degli studi di Milano, Milan, Italy. ${ }^{3}$ Internal Medicine Dept, Respiratory Unit and Adult Cystic Fibrosis Center, Fondazione IRCSS Ca granda Ospedale Maggiore Policlinico, Milan, Italy. ${ }^{4}$ Dept of Pneumology, Hospital Clinic of Barcelona - Institut d'Investigacions Biomèdiques August Pi i Sunyer (IDIBAPS), University of Barcelona, Ciber de Enfermedades Respiratorias (CIBERES), Barcelona, Spain. ${ }^{5}$ Scottish Centre for Respiratory Research, University of Dundee, Dundee, UK.

Correspondence: Aran Singanayagam, Academic Clinical Lecturer in Respiratory Medicine, St Mary's Hospital, Paddington, London W2 1NY, UK. E-mail: aransingadgmail.com

@ERSpublications

Severity scores do not accurately predict response to macrolide therapy in community-acquired pneumonia http://ow.ly/IQjp30dRm7u

Cite this article as: Singanayagam A, Aliberti S, Cillóniz C, et al. Evaluation of severity score-guided approaches to macrolide use in community-acquired pneumonia. Eur Respir J 2017; 50: 1602306 [https:// doi.org/10.1183/13993003.02306-2016].

ABSTRACT International guidelines including those in the UK, Japan, Australia and South Africa recommend the avoidance of macrolides in patients with low-severity community-acquired pneumonia (CAP). We hypothesised that severity scores are poor predictors of atypical pneumonia and response to macrolide therapy, and thus, inadequate tools for guiding antibiotic prescriptions.

Secondary analysis of four independent prospective CAP datasets was conducted. The predictive values of the CURB-65 and pneumonia severity index (PSI) for clinically important groups of causative pathogens were evaluated. The effect of macrolide use according to risk class was assessed by multivariable analysis.

Patients (3297) were evaluated, and the predictive values of CURB-65 and PSI for atypical pathogens were poor (AUC values of 0.37 and 0.42 , respectively). No significant differences were noted among the effects of macrolide use on mortality in patients with mild, moderate and severe CAP, according to either CURB-65 (interaction testing severe versus mild disease OR=0.74 (0.29-1.89)) or PSI (severe versus mild disease $\mathrm{OR}=3.4(0.055-2.10))$, indicating that severity scores were not significant modifiers of response to macrolide therapy.

Severity scores did not accurately predict response to macrolide therapy in CAP, suggesting that current guidance to use these tools for empirical antibiotic choices might not be justified.

This article has supplementary material available from erj.ersjournals.com

Received: Nov 232016 | Accepted after revision: June 082017

Conflict of interest: Disclosures can be found alongside this article at erj.ersjournals.com

Copyright OERS 2017 


\section{Introduction}

Community-acquired pneumonia (CAP) is a major cause of morbidity and mortality worldwide that accounts for a large proportion of antibiotic prescriptions in primary and secondary care [1]. The empirical antibiotic choice is a crucial early management decision for patients presenting with CAP, in view of the fact that microbial aetiology is usually unknown at presentation. International guidelines recommend varying approaches to empirical prescribing, with most countries advocating treatment based on the site of care, and the assumption that the site of care depends on disease severity [2-4]. The most commonly used CAP severity scores, CURB-65 and the pneumonia severity index (PSI), were developed and validated specifically for the prediction of 30-day mortality [5-7]. However, these scores are increasingly being extrapolated to aid other clinical decisions, including the choice of prescribed antibiotics [8].

The British Thoracic Society/National Institute for Health and Care Excellence (BTS/NICE) and other international guidelines, including those in Australia, Japan and South Africa, recommend a specific approach using the CURB-65 score to guide antibiotic choices [9-12]. These recommendations are based on the assumption that low-severity CAP (CURB-65 score 0-1) could be safely treated with oral amoxicillin monotherapy with a broader spectrum cover (including coverage of atypical pathogens with the inclusion of macrolides) reserved for moderate (CURB-65 score 2) or severe (CURB-65 score 3-5) disease. This recommended approach depends on the assumption that atypical coverage with a macrolide is unnecessary in patients with CURB-65 scores of $0-1$, because they are at low risk of an adverse outcome and therefore, coverage of atypical pathogens with macrolide therapy is not essential. These recommendations were based on expert opinions arising from a lack of existing studies from which to draw guidance.

Recently published clinical trials have reported no additional benefit associated with the addition of macrolides to $\beta$-lactam antibiotics as initial empirical CAP therapy $[13,14]$, although one trial $[13]$ has demonstrated beneficial effects in a sub-group with microbiologically confirmed atypical infection. This study also reported no difference in outcome when macrolides were prescribed in low CURB-65 score patients versus those with higher scores [13]. These data provide justification for a formal evaluation of the ability of CURB-65 to predict accurately the presence of atypical infection and response to macrolide therapy, to determine whether a severity score-guided antibiotic approach is appropriate.

The issue of judicious use of macrolide therapy remains extremely important clinically. Macrolides are known to be potent inducers of antibiotic resistance [15] that are associated with important adverse effects, including infections associated with Clostridium difficile [16] and potential cardiotoxicity [17]. Therefore, the use of these drugs must be rigorously justified. Despite the BTS/NICE advocating the use of CURB-65-guided therapy, a previous UK nationwide audit showed that $51 \%$ of patients with low severity CAP, according to CURB-65, received broad-spectrum cover with a $\beta$-lactam in addition to macrolide therapy [18]. This apparent failure of clinicians to adopt the recommended approach further justifies an examination of whether it is appropriate.

The aim of our study was to determine the value of the severity score-based approaches for guidance of antibiotic therapy in patients hospitalised with CAP. We hypothesised that CURB-65 and PSI would be poor predictors of the presence of atypical pathogens, and would also be poorly predictive of response to macrolide therapy, and thus be inadequate tools for guiding appropriate prescription of macrolides in CAP.

\section{Methods}

\section{Study populations}

This study entailed a secondary analysis of four large prospective observational datasets: the Edinburgh Pneumonia Study (Edinburgh, UK; 2005-2010); the Milan Pneumonia Study (Policlinico Hospital, Milan, Italy; 2008-2010); the Failure and Cardiovascular Events in Community-acquired Pneumonia (FAILCAP) dataset (an Italian multicentre study; 2009-2012); and the Barcelona pneumonia cohort (Barcelona, Spain; 2008-2012). All four studies enrolled consecutive and unselected adult patients who were hospitalised with radiographically confirmed CAP. Details for each study have been previously published [19-22]. Inclusion and exclusion criteria for the four studies are detailed in the online supplementary methods.

\section{Data collection}

In all four cohorts, demographic variables, baseline admission observations, standard blood tests (full blood count, urea and electrolytes, liver function tests, albumin and C-reactive protein) and initial therapies, including antibiotics, were measured and recorded on hospital admission. Patients were risk-assessed on admission, using the CURB-65 score [5] and the PSI [6]. In the Barcelona cohort, only the PSI could be calculated. 


\section{Microbiological evaluation}

Microbiological testing was conducted according to BTS recommendations in the Edinburgh cohort [9], and according to the European Respiratory Society recommendations in the Milan, FAILCAP and Barcelona cohorts [2]. Admitting clinicians were responsible for decisions regarding microbiological testing. Sputum and blood cultures were recommended within $24 \mathrm{~h}$ of hospital admission. Urinary antigen testing was performed for Legionella pneumophila in all three cohorts, and for Streptococcus pneumoniae in the Milan cohort alone. Pleural puncture, tracheobronchial aspirates, and bronchoalveolar lavage fluid, when available, were also collected and cultured. Identification of microorganisms and susceptibility testing were performed according to standard methods. Identification of atypical pathogens by PCR was carried out in all four cohorts, and atypical serology was carried out only in the Milan and FAILCAP cohorts. The online supplementary material gives detailed information regarding atypical testing. Multidrug-resistant pathogens were defined as previously described [20].

\section{Statistical analyses}

All data were analysed and processed using the SPSS version 21.0 for Windows (SPSS Inc., Chicago, Ill, USA). Descriptive statistics of demographic and clinical variables were expressed as median (interquartile range (IQR)), unless otherwise stated. The area under the receiver operating characteristic curve (AUC) was used to assess predictive tests.

Multivariable logistic regression was used to test the effect of antibiotic therapies on 30-day mortality after adjustment for relevant confounders (all variables included in PSI, except altered mental status, urea, respiratory rate, blood pressure and age in the case of CURB-65 and multilobar radiographic changes, and the presence of severe sepsis on admission in the case of PSI). To test if severity scores modified the effects of antibiotics on mortality, odds ratios for mortality between the mild, moderate and severe groups were compared, using interaction testing as described by Altman and Bland [23]. For all analyses, a two-tailed p-value $<0.05$ was considered statistically significant.

\section{Results}

A total of 790 patients from the Edinburgh pneumonia study, 935 patients from the Milan cohort, 667 patients from the Barcelona pneumonia study and 905 patients from the FAILCAP cohort were included in the present study, giving an overall combined total cohort of 3297 patients. Table 1 shows baseline characteristics of the three cohorts separately, and the overall combined cohort. Table 2 shows the frequency of identified pathogens in each cohort separately, and in the combined cohort.

\section{Frequency of pathogens according to CURB-65 or PSI risk class}

Table 3 shows the frequency of pathogens in severe versus non-severe risk classes according to CURB-65, with risk ratios showing a comparison of frequencies between severe and non-severe classes. The frequency of Mycoplasma pneumoniae and all atypical pathogens combined was significantly higher in patients with non-severe disease according to both CURB-65 and PSI. The frequency of L. pneumophila was significantly higher in patients with non-severe disease, according to CURB-65. The frequency of Gram-negative Enterobacteriaceae was significantly higher in patients with severe disease compared to those with non-severe disease, according to both CURB-65 and PSI. Methicillin-sensitive Staphylococcus aureus (MSSA) was significantly more frequent in patients with severe disease according to CURB-65 and PSI, whereas methicillin-resistant Staphylococcus aureus (MRSA) and multidrug-resistant (MDR) pathogens combined were significantly more frequent in patients with severe disease, according to the PSI. The frequency of pathogens according to CURB-65 and PSI in each of the individual cohorts is shown in supplementary tables S1 and S2.

\section{Predictive value of severity scores to determine causative pathogens in CAP}

The predictive value of severity scores according to CURB-65 and PSI, for clinically relevant groups of pathogens was evaluated. Table 4 shows the AUC for prediction of atypical bacteria, MSSA and MRSA, Gram-negative Enterobacteriaceae, Pseudomonas aeruginosa and MDR pathogens. Both severity scores performed poorly for the prediction of all pathogen groups, with AUC values consistently less than 0.7 (the minimum threshold regarded for a clinically useful test). The AUC value for the prediction of atypical bacteria was $<0.5$ for both CURB-65 and PSI, indicating that the use of these scores could provide misleading information.

\section{Macrolide use and mortality according to admission severity score in CAP}

The effect of macrolide use on mortality, stratified according to the admission severity score, is shown in table 5. Although macrolide use was significantly associated with reduced mortality in severe disease, but not in moderate or mild disease according to CURB-65, severity criteria were not considered modifiers of 
TABLE 1 Baseline characteristics of individual cohorts and overall combined dataset

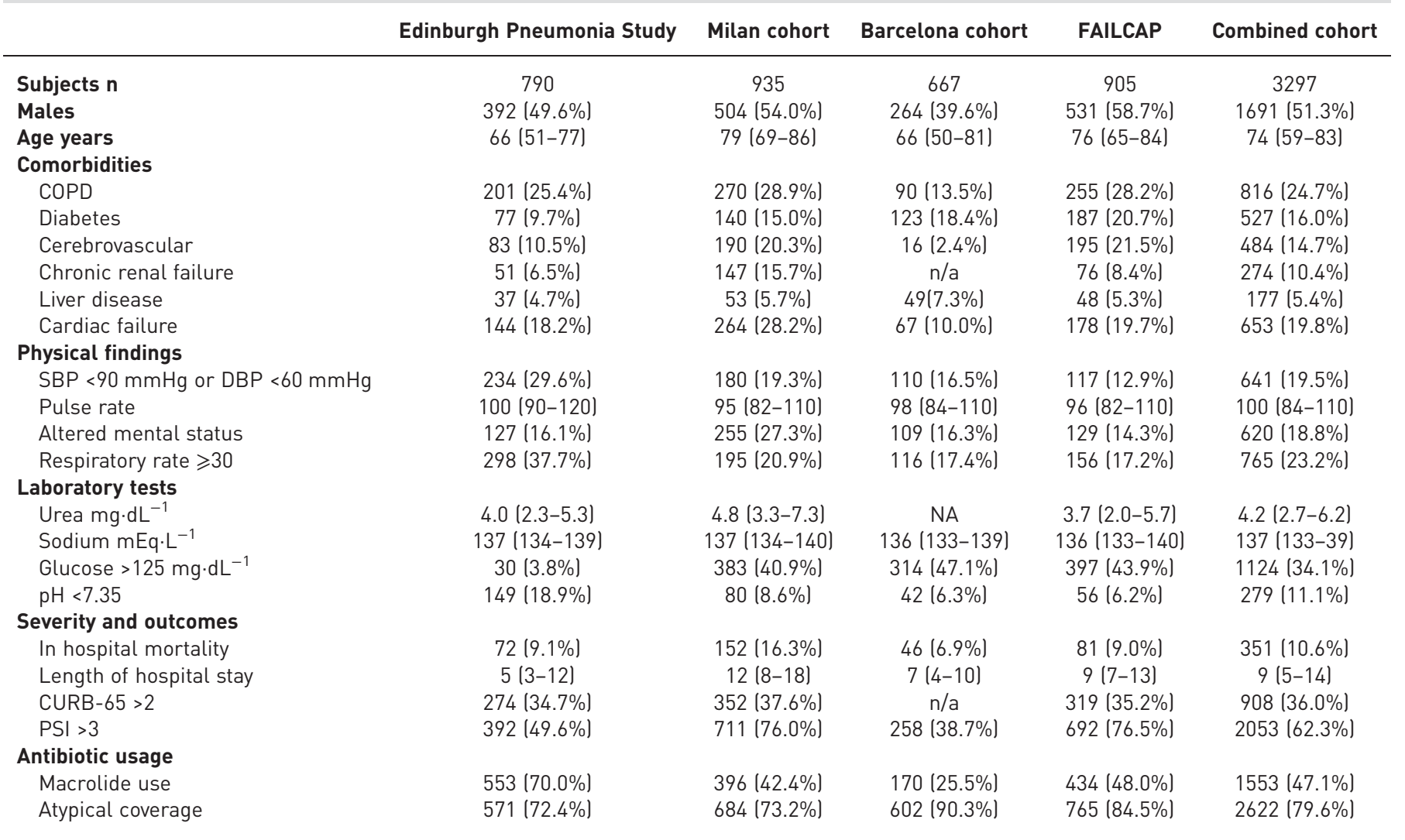

Data are presented as $\mathrm{n}(\%)$ or median (interquartile range, unless otherwise stated. FAILCAP: Failure and Cardiovascular Events in Community-acquired Pneumonia; COPD: chronic obstructive pulmonary disease; SBP: systolic blood pressure; DBP: diastolic blood pressure; PSI: pneumonia severity index.

the benefits of macrolide use. This is because no significant differences were noted between the effects of macrolide use on mortality, when patients with mild, moderate and severe disease according to CURB-65, were compared by interaction testing (see table 6). Similar observations were noted for the PSI, except for severe versus moderate disease, for which there was a significantly greater effect on mortality in the

TABLE 2 Pathogen frequency in individual cohorts and combined dataset

\begin{tabular}{|c|c|c|c|c|c|}
\hline & Edinburgh cohort & Milan cohort & Barcelona cohort & FAILCAP & Combined cohort \\
\hline Subjects $n$ & 790 & 935 & 667 & 905 & 3297 \\
\hline $\begin{array}{l}\text { n (\% of patients with positive } \\
\text { pathogen identified) }\end{array}$ & $224(28.3 \%)$ & $170(18.2 \%)$ & $181(27.1 \%)$ & $145(16.0 \%)$ & $720(21.8 \%)$ \\
\hline Streptococcus pneumoniae & $107(47.8 \%)$ & $63(37.1 \%)$ & $123(68.0 \%)$ & $54(37.2 \%)$ & $347(48.2 \%)$ \\
\hline Haemophilus influenzae & $22(9.8 \%)$ & $6(3.5 \%)$ & $5(2.7 \%)$ & $3(2.1 \%)$ & $36(5.0 \%)$ \\
\hline Mycoplasma pneumoniae & $13(5.8 \%)$ & $5(2.9 \%)$ & $3(1.7 \%)$ & $8(5.5 \%)$ & $29(4.0 \%)$ \\
\hline MRSA & $2(0.9 \%)$ & $16(9.4 \%)$ & $10(5.5 \%)$ & $12(8.3 \%)$ & $40(5.6 \%)$ \\
\hline Klebsiella pneumoniae & $10(4.5 \%)$ & $13(7.6 \%)$ & $3(1.7 \%)$ & $2(1.4 \%)$ & $28(3.9 \%)$ \\
\hline Chlamydia pneumoniae & $1(0.4 \%)$ & $4(2.4 \%)$ & $0(0 \%)$ & $2(1.4 \%)$ & $7(1.0 \%)$ \\
\hline All atypicals combined & $22(9.8 \%)$ & $35(20.6 \%)$ & $15(8.3 \%)$ & $29(20.0 \%)$ & $101(14.0 \%)$ \\
\hline Gram-negative Enterobacteriaceae & $15(6.7 \%)$ & $30(17.6 \%)$ & $10(5.5 \%)$ & $13(9.0 \%)$ & $68(9.4 \%)$ \\
\hline Pseudomonas aeruginosa & $2(0.9 \%)$ & $12(7.1 \%)$ & $13(7.2 \%)$ & $7(4.8 \%)$ & $34(4.7 \%)$ \\
\hline MDR pathogens combined & $7(3.1 \%)$ & $58(34.1 \%)$ & $12(6.6 \%)$ & $18(12.4 \%)$ & $95(13.2 \%)$ \\
\hline
\end{tabular}

FAILCAP: Failure and Cardiovascular Events in Community-acquired Pneumonia; MSSA: methicillin-sensitive Staphylococcus aureus; MRSA: methicillin-resistant Staphylococcus aureus, MDR: multidrug-resistant. 
TABLE 3 Pathogen frequency according to admission severity class

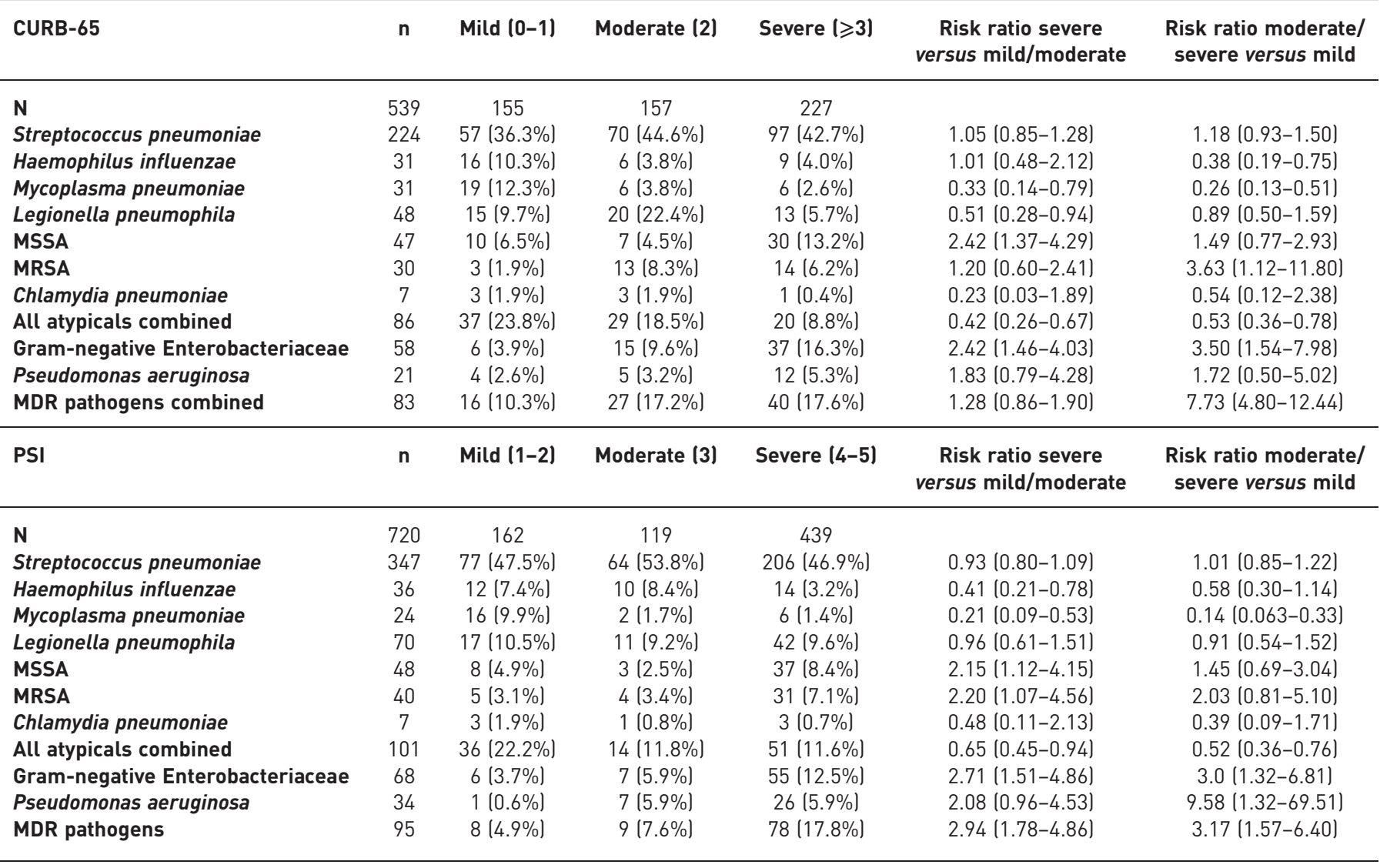

MSSA: methicillin-sensitive Staphylococcus aureus; MRSA: methicillin-resistant Staphylococcus aureus; MDR: multidrug-resistant; PSI: pneumonia severity index.

moderate severity group. These findings indicate that existing severity scores are not significant modifiers of response to macrolide use.

Macrolide use in subgroups with confirmed atypical pathogens and non-atypical pathogens There were 101 patients with confirmed atypical pathogens in the combined cohort. Of these patients, 57 received macrolide therapy. In the sub-group with confirmed atypical pathogens, the unadjusted effect of macrolide use (either monotherapy or combination therapy) showed no significant effect on mortality (OR 0.48, 95\% CI 0.21-1.05); however, this became significant after adjustment for admission severity, according to PSI (OR 0.36, 95\% CI 0.13-0.99).

In the sub-group with evidence of typical pathogens only, macrolide use (either monotherapy or combination therapy) was also significantly associated with reduced mortality (OR 0.60, 95\% CI 0.43-0.84), an effect that remained significant after adjustment for admission severity (OR 0.47, 95\% CI 0.30-0.74).

TABLE 4 Predictive value of severity scores for clinically important groups of pathogens in community-acquired pneumonia

\begin{tabular}{lcccccc} 
& Atypical bacteria & Gram-negative Enterobacteriaceae & MSSA & MRSA & Pseudomonas & All MDR pathogens \\
\hline CURB-65 & $0.37(0.31-0.43)$ & $0.65(0.58-0.72)$ & $0.61(0.52-0.69)$ & $0.57(0.48-0.67)$ & $0.58(0.46-0.71)$ & $0.56(0.49-0.62)$ \\
PSI & $0.42(0.35-0.48)$ & $0.61(0.55-0.68)$ & $0.58(0.50-0.66)$ & $0.69(0.50-0.67)$ & $0.60(0.52-0.69)$ & $0.63(0.57-0.68)$ \\
\hline
\end{tabular}

MSSA: methicillin-sensitive Staphylococcus aureus; MRSA: methicillin-resistant Staphylococcus aureus; MDR: multidrug-resistant; PSI: pneumonia severity index. 
TABLE 5 Association between macrolide use and mortality, stratified according to severity

CURB-65

Pneumonia severity index

Mild

Unadjusted

Adjusted

Moderate

Unadjusted

Adjusted

Severe

Unadjusted

Adjusted

$0.47(0.21-1.06)$
$0.51(0.21-1.21)$
$0.66(0.43-1.02)$
$0.60(0.36-1.01)$
$0.67(0.53-0.86)$
$0.69(0.49-0.97)$

$0.57(0.45-0.73)$

$0.44(0.31-0.61)$

$0.470 .35-0.64$

$0.35(0.24-0.53)$

$0.62(0.53-0.72)$

$0.52(0.42-0.64)$

Data are presented as odds ratios with $95 \%$ confidence intervals.

Sensitivity analysis excluding patients in whom atypical pathogens were diagnosed solely by serological tests

As previous studies have demonstrated that serology for Chlamydia pneumoniae and M. pneumoniae (with the exception of the IgM antibody) is not accurate for the diagnosis of atypical pneumonia [24, 25], we performed a sensitivity analysis in which atypical pneumonia cases that were identified by serology alone were excluded (only present in the Barcelona cohort and thus, only relevant for analysis of the PSI). A consistent effect was observed in this sub-group, with the frequency of atypical pathogens combined being significantly higher in patients with non-severe disease versus those with severe disease, according to the PSI (risk ratio $0.51,95 \%$ CI $0.35-0.75$ ). The AUC for the prediction of atypical bacteria based on the PSI, after exclusion of cases diagnosed by serology alone, was 0.39 (95\% CI $0.32-0.46)$.

\section{Discussion}

Our study of a large international cohort, comprising four independent European datasets, demonstrates that international guidance from the UK, Japan, Australia and South Africa on the use of admission severity scoring for empirical antibiotic prescribing in CAP, specifically to determine the use of macrolides, is unjustified. This conclusion is based on our finding that severity scores, such as the CURB-65 and PSI, are not predictors of response to macrolide therapy and do not accurately predict the presence of atypical pathogens.

We demonstrated that severity scores, such as CURB-65 and PSI, are poor predictors of microbial aetiology, with AUC values consistently below 0.7 , the minimum threshold above which predictive scores can be deemed clinically useful [26]. In particular, we showed that severity is not a reliable predictor of the presence of atypical pathogens. Remarkably, in the present study, CURB-65 had an AUC of 0.37 for the prediction of atypical microorganisms, indicating that atypical pathogens were actually more frequent in the low-severity classes of CURB-65, and that a coin toss (giving an AUC of 0.5) would be more predictive of atypical pathogens. The more detailed score, the PSI, had a similarly low AUC value of 0.42 .

TABLE 6 Interaction testing of different severity classes according to either CURB-65 or pneumonia severity index (PSI) for effect of macrolide use on mortality

\begin{tabular}{lccc} 
Macrolide use & Ratio of relative risk & Z score & $\mathbf{9 5 \%} \mathbf{C l}$ \\
\hline $\begin{array}{l}\text { Severe versus mild } \\
\text { CURB-65 }\end{array}$ & 0.74 & & \\
PSI & 0.34 & -0.63 & $0.29-1.89$ \\
Moderate versus mild & & -1.16 & $0.055-2.10$ \\
$\quad$ CURB-65 & 0.85 & -0.31 & \\
PSI & 0.80 & -0.86 & $0.30-2.35$ \\
Severe versus moderate & & & $0.47-1.34$ \\
CURB-65 & 1.15 & 0.38 & $0.55-2.39$ \\
PSI & 0.61 & -2.06 & $0.29-0.98$
\end{tabular}

Interaction testing performed as previously described by ALTMAN and BLAND [23]. Relative risk ratio <1: lesser relative benefit for first group stated; relative risk ratio $>1$ : greater benefit for first group stated. 
The current approach advocated by the UK, Japanese, Australian and South African pneumonia guidelines is based on the premise that narrow spectrum therapy (with a lack of atypical cover) is adequate for patients with low-severity disease, with broader spectrum therapy (including coverage of atypical pathogens and $S$. aureus) being reserved for patients in higher severity classes. These guidelines recommend that macrolide therapy should not be administered to patients with low admission severity CAP (CURB-65 score of 0-1). Given our findings that atypical pathogens occur frequently in this group, this approach would be expected to lead to inadequate coverage of atypical bacteria in a considerable proportion of patients. These concerns are reinforced by previous studies that have also evaluated microbial aetiology according to the severity of CAP, and have similarly shown that atypical pathogens are more frequent in lower severity classes [27-29]. Expert opinion has previously raised concerns over inadequate coverage of atypical microorganisms in hospitalised patients with low severity disease, as recommended by such strategies [30]. A counter-argument to this theory would be that inadequate pathogen coverage in patients with milder disease is less likely to be harmful and might simply require an adjustment in the treatment plan. However, in a study focusing on Legionella pneumonia by vON BAUM et al. [31], 37.5\% of patients who died within 6 months received antibiotics that did not initially cover atypical microorganisms after initially presenting with mild pneumonia. Therefore, this would suggest that withholding macrolide therapy might not be appropriate for all patients with mild pneumonia, based exclusively on severity scores.

Regardless of whether or not severity scores can accurately predict microbial aetiology, it could be argued that the benefits of macrolide therapy are not solely related to antibacterial activity, but might also be related to the widely reported anti-inflammatory effects of these drugs [32]. Previous studies have shown that even in patients with confirmed non-atypical (pneumococcal) infection, the addition of macrolide therapy has beneficial effects $[33,34]$. Although some observational studies have historically reported a benefit associated with macrolide use in CAP [35], two recent randomised controlled trials have disproved this, and have shown no additional benefit associated with the addition of macrolides to $\beta$-lactam empirical therapy in hospitalised patients with CAP $[13,14]$. Despite these reported findings, specific subgroups of patients that derive benefit from macrolides might still exist. A severity score-guided approach would still be justifiable if severity scores could be shown to predict macrolide treatment response accurately with minimal beneficial effects in patients with low CURB-65 scores. However, the findings of our study also contradict this assertion, because we found no significant differences between the effects of macrolide use on mortality, when patients with mild, moderate and severe disease according to CURB-65 were compared by interaction testing, thereby demonstrating that severity criteria are not modifiers of the benefits of macrolide use. A previous study by RODRIGo et al. [36] evaluated 30-day mortality in patients treated with combined $\beta$-lactam/macrolide therapy versus $\beta$-lactam therapy alone, and concluded that combination therapy was associated with lower inpatient mortality, specifically in patients with moderate and severe CAP according to CURB-65, but not in those with low severity CAP. However, the authors did not apply interaction testing to their data, which would have shown a result consistent with our finding of no differences in the effects of macrolide use on mortality in mild versus severe pneumonia according to CURB-65.

Our findings mirror those in the trial reported by GARIN et al. [13], which reported no significant benefits of combination therapy to achieve the primary outcome of reaching clinical stability at day 7. It should be noted, however, that patients with positive urinary antigen for Legionella were excluded from this study, and their inclusion might have led to the manifestation of a greater effect. Although macrolide use was shown to confer greater benefit to the group with severe disease according to the PSI versus those with low PSI scores, no difference was observed in the outcomes when the cohort was stratified according to CURB-65 [13]. When combined with our findings, these data argue strongly against the use of CURB-65 as a tool for guiding the use of macrolides in clinical practice, and suggest reconsideration of the current international guidelines.

The aforementioned study by GARIN et al. [13] also reported that macrolide use increased the achievement of clinical stability at day 7 in patients with confirmed atypical infection, but not in those with non-atypical infection, suggesting that the presence of atypical pathogens is an important factor in determining macrolide response. Given our finding that atypical pathogens occur most frequently in patients with low CURB-65 scores, it seems counterintuitive to deny these patients macrolide therapy. It should be noted that our study found a beneficial effect of macrolide use on mortality both in patients with atypical and non-atypical infection. The apparent discordance with the GARIN et al. [13] study could be explained by the presence of inter-cohort variability in the atypical testing conducted in the cohorts included in the present study. There was a 2 -fold increase in the frequency of atypical pathogens observed in the Milan and FAILCAP cohorts, based in Italy, most likely reflecting the increased use of atypical serology testing employed at these centres. Therefore, it is possible that some patients in our combined 
cohort might have been misclassified as having no atypical infection because of a lack of appropriate testing. This might account for the differences between our observational study and those of the randomised controlled trial by GARIN et al. [13], in which standardised microbiological testing was adopted.

Macrolide use in CAP has been a subject of growing interest and judicious use of these therapies is strongly advocated because of emerging concerns regarding complications associated with over-use, such as infections associated with $C$. difficile and the development of antibiotic-resistant strains $[15,16,37]$. Severity score-guided approaches are commendable, in that they are geared towards limiting macrolide over-use, and thus minimising associated risks. However, the data from our study would suggest that using CURB-65 as a method to determine which patients should receive macrolide therapy is unjustified. Despite the fact that CURB-65-guided therapy is clearly advocated by the BTS/NICE guidelines, a previous nationwide audit of CAP management in the UK reported poor compliance with these recommendations [18]. Over 50\% of patients with low CURB-65 scores were empirically prescribed combination $\beta$-lactam/macrolide therapy in contradiction with the recommended approach of targeting such therapy at patients with severe disease only. Studies are now required to determine the specific subgroups of patients with CAP who derive clinical benefit from the use of macrolides, to inform practice and future guidance.

Despite the fact that PSI and CURB-65 were shown to be poor predictors of microbial aetiology in the present study, statistically significant associations were observed between the severity scores evaluated and the presence of Gram-negative Enterobacteriaceae, S. aureus, and MDR pathogens. This finding emphasises and supports international guideline recommendations to provide Gram-negative and Staphylococcal coverage to patients with a high severity of illness [9]. To date, only one study has evaluated a CURB-65-guided antibiotic therapy strategy in a clinical setting [38]. That study compared antibiotic prescribing and outcomes before and after implementation of the strategy in a tertiary care centre. The post-intervention group showed a significant reduction in broad-spectrum antibiotic prescription, without any corresponding effect on mortality. Although macrolide use was reduced post-intervention, it remained relatively high, even in patients with low-severity disease. Although that study suggested that a CURB-65 guided antibiotic approach was a safe strategy in a hospitalised cohort, no other independent validation of these findings has been conducted, and it is important to note that this was a before-and-after evaluation rather than a carefully matched randomised controlled trial.

The present study was not designed to answer whether empirical macrolide therapy is effective in CAP. This question can only be answered through large randomised controlled trials. Rather, our study was designed to evaluate whether the evidence supports the use of a severity scoring system, to decide which patients should be administered a macrolide. Our study demonstrates that CURB-65 does not predict various populations that have different levels of macrolide response. Alternative severity scores, such as the Japanese Respiratory Society guidelines, which have been evaluated for the prediction of Mycoplasma pneumonia [39], might be more effective than CURB-65 at guiding appropriate macrolide therapy; however, further studies are needed.

Our study should not be interpreted as a call to treat all hospitalised CAP patients with macrolides, but rather to remove an imperfect scoring system from the decision-making process, and focus exclusively on a balance between the existing evidence for macrolide treatment and the risks of macrolide treatment, such as antimicrobial resistance and drug-related adverse effects. This balance might vary in individual patients, or in different healthcare environments.

The strengths of the present study include the prospective collection of data and the demonstration of the validity of our findings in four independent cohorts from Northern and Southern Europe, with different empirical antibiotic prescribing practices, microbiological testing regimes and healthcare systems. This suggests that our data are robust, and likely to be applicable to similar healthcare settings internationally. Nevertheless, limitations of the analysis must also be acknowledged. The frequency of positive microbiological results was low. However, this is similar to the findings of other cohorts that have been reported internationally, and reflects the limitations of the currently available microbiological tests for CAP. In addition, in all four cohorts, microbiological testing was left to the discretion of the admitting physician, but was carried out in accordance with international guidelines. As stated previously, we cannot exclude the occurrence of false negative results when a microorganism has not been identified because of the lack of appropriate testing. Future studies using a more systematic approach to atypical testing in all patients, regardless of admission severity would provide clarity. It should also be noted that our study considered only hospitalised patients, and further research is needed to determine the value of severity scores to guide antibiotic choices in patients treated within the community. 
In conclusion, our study demonstrates that severity scores cannot be used to accurately identify subgroups of patients with CAP with different macrolide responses. Further evidence of impact is required before severity score-guided regimes can be recommended for empirical antibiotic prescribing in CAP.

\section{References}

1 National Institute for Health and Care Excellence (NICE). Respiratory tract infections - prescribing antibiotics. 2008. www.nice.org.uk/nicemedia/pdf/CG69FullGuideline.pdf Last accessed: April 2013.

2 Woodhead M, Blasi F, Ewig S, et al. Guidelines for the management of adult lower respiratory tract infections--full version. Clin Microbiol Infect 2011; 17: Suppl. 6, E1-59.

3 Mandell LA, Wunderink RG, Anzueto A, et al. Infectious Diseases Society of America/American Thoracic Society consensus guidelines on the management of community-acquired pneumonia in adults. Clin Infect Dis 2007; 44: Suppl. 2, S27-S72.

4 Menéndez R, Torres A, Aspa J, et al. Neumonía adquirida en la comunidad. Nueva normativa de la Sociedad Española de Neumología y Cirugía Torácica (SEPAR). [Community acquired pneumonia. New guidelines of the Spanish Society of Chest Diseases and Thoracic Surgery (SEPAR).] Arch Bronconeumol 2010; 46: 543-558.

5 Lim WS, van der Eerden MM, Laing R, et al. Defining community acquired pneumonia severity on presentation to hospital: an international derivation and validation study. Thorax 2003; 58: 377-382.

6 Fine MJ, Auble TE, Yealy DM, et al. A prediction rule to identify low-risk patients with community-acquired pneumonia. N Engl J Med 1997; 336: 243-250.

7 Chalmers JD, Singanayagam A, Akram AR, et al. Severity assessment tools for predicting mortality in hospitalised patients with community-acquired pneumonia. Systematic review and meta-analysis. Thorax 2010; 65: 878-883.

8 Singanayagam A, Chalmers JD. Severity assessment scores to guide empirical use of antibiotics in community acquired pneumonia. Lancet Respir Med 2013; 1: 653-662.

9 Lim WS, Baudouin SV, George RC, et al. BTS guidelines for the management of community acquired pneumonia in adults: update 2009. Thorax 2009; 64: Suppl. 3, iiil-ii55.

10 Yanagihara K, Kohno S, Matsusima T. Japanese guidelines for the management of community-acquired pneumonia. Int J Antimicrob Agents 2001; 18: Suppl. 1, S45-S48.

11 Therapeutic Guidelines Limited. Therapeutic guidelines: Antibiotic. Version 14. Melbourne, Therapeutic Guidelines Limited, 2010.

12 Working Group of the South African Thoracic Society. Management of community-acquired pneumonia in adults. S Afr Med J 2007; 97: 1296-1306.

13 Garin $\mathrm{N}$, Genné $\mathrm{D}$, Carballo $\mathrm{S}$, et al. $\beta$-Lactam monotherapy vs $\beta$-lactam-macrolide combination treatment in moderately severe community-acquired pneumonia: a randomized noninferiority trial. JAMA Intern Med 2014; 174: 1894-1901.

14 Postma DF, van Werkhoven $\mathrm{CH}$, van Elden LJ, et al. Antibiotic treatment strategies for community-acquired pneumonia in adults. N Engl J Med 2015; 372: 1312-1323.

15 Principi N, Esposito S. Macrolide-resistant Mycoplasma pneumoniae: its role in respiratory infection. J Antimicrob Chemother 2013; 68: 506-511.

16 Brown KA, Khanafer N, Daneman N, et al. Meta-analysis of antibiotics and the risk of community-associated Clostridium difficile infection. Antimicrob Agents Chemother 2013; 57: 2326-2332.

17 Schembri S, Williamson PA, Short PM, et al. Cardiovascular events after clarithromycin use in lower respiratory tract infections: analysis of two prospective cohort studies. BMJ 2013; 346: f1235.

18 Lim WS, Woodhead M. British Thoracic Society adult community acquired pneumonia audit 2009/10. Thorax 2011; 66: 548-549.

19 Singanayagam A, Singanayagam A, Chalmers JD. Obesity is associated with improved survival in community-acquired pneumonia. Eur Respir J 2013; 42: 180-187.

20 Aliberti S, Di Pasquale M, Zanaboni AM, et al. Stratifying risk factors for multidrug-resistant pathogens in hospitalized patients coming from the community with pneumonia. Clin Infect Dis 2012; 54: 470-478.

21 Aliberti S, Ramirez J, Consentini R, et al. Acute myocardial infarction versus other cardiovascular events in community-acquired pneumonia. ERJ Open Res 2015; 1: 00020-2015.

22 Cilloniz C, Albert RK, Liapikou A, et al. The effect of macrolide resistance on the presentation and outcome of patients hospitalized for Streptococcus pneumoniae pneumonia. Am J Respir Crit Care Med 2015; 191: 1265-1272.

23 Altman DG, Bland JM. Interaction revisited: the difference between two estimates. BMJ 2003; 326: 219.

24 von Baum $\mathrm{H}$, Welte $\mathrm{T}$, Marre $\mathrm{R}$, et al. Mycoplasma pneumoniae pneumonia revisited within the German Competence Network for Community-acquired pneumonia (CAPNETZ). BMC Infect Dis 2009; 9: 62.

25 Wellinghausen N, Straube E, Freidank H, et al. Low prevalence of Chlamydia pneumoniae in adults with community-acquired pneumonia. Int J Med Microbiol 2006; 296: 485-491.

26 Singanayagam A, Chalmers JD, Hill AT. Severity assessment in community-acquired pneumonia: a review. QJM 2009; 102: 379-388

27 Strålin K, Olcén P, Törnqvist E, et al. Definite, probable, and possible bacterial aetiologies of community-acquired pneumonia at different CRB-65 scores. Scand J Infect Dis 2010; 42: 426-434.

28 Cillóniz C, Ewig S, Polverino E, et al. Microbial aetiology of community-acquired pneumonia and its relation to severity. Thorax 2011; 66: 340-346.

29 Rosón B, Carratalà J, Dorca J, et al. Etiology, reasons for hospitalization, risk classes, and outcomes of community-acquired pneumonia in patients hospitalized on the basis of conventional admission criteria. Clin Infect Dis 2001; 33: 158-165.

30 Torres A, Rello J. Update in community-acquired and nosocomial pneumonia 2009. Am J Respir Crit Care Med 2010; 181: 782-787.

31 von Baum H, Ewig S, Marre R, et al. Community-acquired Legionella pneumonia: new insights from the German competence network for community acquired pneumonia. Clin Infect Dis 2008; 46: 1356-1364.

32 Zarogoulidis P, Papanas N, Kioumis I, et al. Macrolides: from in vitro anti-inflammatory and immunomodulatory properties to clinical practice in respiratory diseases. Eur J Clin Pharmacol 2012; 68: 479-503. 
33 Martínez JA, Horcajada JP, Almela M, et al. Addition of a macrolide to a beta-lactam-based empirical antibiotic regimen is associated with lower in-hospital mortality for patients with bacteremic pneumococcal pneumonia. Clin Infect Dis 2003; 36: 389-395.

34 Baddour LM, Yu VL, Klugman KP, et al. Combination antibiotic therapy lowers mortality among severely ill patients with pneumococcal bacteremia. Am J Respir Crit Care Med 2004; 170: 440-444.

35 Nie W, Li B, Xiu Q. $\beta$-Lactam/macrolide dual therapy versus $\beta$-lactam monotherapy for the treatment of community-acquired pneumonia in adults: a systematic review and meta-analysis. J Antimicrob Chemother 2014; 69: 1441-1446.

36 Rodrigo C, McKeever TM, Woodhead M, et al. Single versus combination antibiotic therapy in adults hospitalised with community acquired pneumonia. Thorax 2013; 68: 493-495.

37 Chalmers JD, Akram AR, Singanayagam A, et al. Risk factors for Clostridium difficile infection in hospitalized patients with community-acquired pneumonia. J Infect 2016; 73: 45-53.

38 Chalmers JD, Singanayagam A, Akram AR, et al. Safety and efficacy of CURB65-guided antibiotic therapy in community-acquired pneumonia. J Antimicrob Chemother 2011; 66: 416-423.

39 Yin YD, Zhao F, Ren LL, et al. Evaluation of the Japanese Respiratory Society guidelines for the identification of Mycoplasma pneumoniae pneumonia. Respirology 2012; 17: 1131-1136. 